\title{
Self-insertion of a screwdriver into the rectum for sexual pleasure: A case report
}

\author{
Mohammad Reza Sharif MD and Javad Alizargar MD \\ Affiliation: \\ Kashan University of Medical Sciences, Kashan, I.R. Iran
}

\begin{abstract}
Background

Rectal foreign bodies are rare conditions that happen mostly for sexual purposes. Sex toys are the commonest objects. A 56 year old man came to the emergency department of the Shahid Beheshti Hospital with pain in the left lower quadrant of his abdomen and a feeling of discomfort in the anal area. Digital rectal examination was normal but a metal object was palpable $8 \mathrm{~cm}$ distal to the anal verge. A plain x-ray demonstrated a screwdriver in the rectum. Initial attempts at manual extraction were unsuccessful in the emergency department. An attempt at removing the screwdriver from the rectum using Kelly Forceps with the patient in the jackknife position was successful.
\end{abstract}

\section{Keywords}

Foreign body, rectum, emergency department, emergency medicine

\section{INTRODUCTION}

Rectal foreign bodies are rare conditions that are more frequent in men, usually aged $30-40$ years, but have been reported in all ages. A variety of foreign bodies have been reported in the literature, ranging from dildos and vibrators to unusual objects like turkey basters (1). There are voluntary versus involuntary and sexual versus non-sexual types of foreign bodies, but the most common type is voluntary insertion of an object for sexual stimulation. The nature of this type of emergency necessitates a careful physical examination and history taking by emergency doctors, and it will be highlighted when we consider the shame of the patient talking about their condition (1). There may be no foreign object present at the time of arrival of the patient due to successful removal of the object, but perforation, bleeding or incontinence may be the only symptoms a patient has (2). First approach to foreign objects in the rectum in the absence of anorectal lacerations or injuries may be the removal via the per-anum approach. But in complicated cases or failure of per-anum removal of the object laparoscopy surgery may be inevitable (3).
We report the first case of self-penetration of a screwdriver in the rectum for sexual stimulation and the trans-anal removal of this object at the Shahid Beheshti Hospital.

\section{CASE REPORT}

A 56 year old man came to the emergency department of Shahid Beheshti hospital in the early morning. His chief complaint was pain in left lower quadrant of his abdomen. He had a history of hospitalisation 20 years ago due to Post Traumatic Stress Disorder (PTSD) after the war of Iran and Iraq. He has not been on any medication in recent years. His vital signs were stable, heart rate of 72 , respiratory rate of 18 and blood pressure of $125 / 85$. He had a light general tenderness of the abdomen and a feeling of discomfort in the anal area. Digital rectal examination was normal and no bloody or tarcoloured discharge was observed. However, a metal object was palpable approximately $8 \mathrm{~cm}$ from the anal verge. Bowel sounds were normal with no evidence of internal haemorrhage, perforation, or peritonitis. A plain $\mathrm{x}$-ray demonstrated a screwdriver in the rectum, see 
Figure 1. After a consult with a psychologist the patient admitted that after sexual arousal by a pornographic video, he deliberately inserted a screwdriver into his anus approximately six hours earlier. He did it so profoundly and couldn't retrieve it. In the emergency department the screwdriver could not be removed digitally. After spinal anaesthesia, an attempt at removing the screwdriver using Kelly Forceps with the patient in the jackknife position was successful with a $32 \mathrm{~cm}$ screwdriver removed from the rectum. After post removal recto-sigmoidoscopy and ruling out bowel injury, the patient was observed for two days in the surgery ward and referred to the psychology hospital for further evaluation and management.

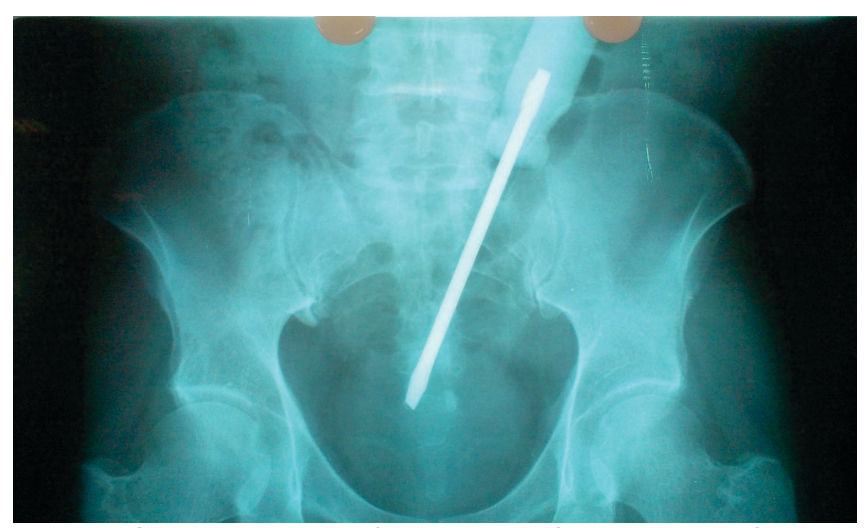

Figure 1: Simple pelvic $x$-ray for diagnosis of the presence of foreign body in the rectum

\section{DISCUSSION}

In western countries the insertion of various vibrators into the rectum for sexual purposes is the most common (up to $50 \%$ ) cause of retained rectal foreign bodies (4). But in countries such as Iran, the limited availability of these kinds of sexual arousal objects, and the taboo nature of such actions, it is not frequently seen in the emergency department. Therefore, the patient may not be inclined to present to a hospital emergency department and hence this lack of action may lead to further worsening of the situation. In this case the patient trying to manually remove the object may have resulted in the object moving further up the rectum towards the sigmoid colon and making the situation more complicated. The patient has a history of inserting objects in his rectum for sexual purposes, and this time he chose a screwdriver for more satisfaction. Although a lack of judgment in choosing a screwdriver may be the main cause of choosing an unusual and dangerous tool, especially when we look at his psychological background, the unavailability of sexual toys may be another reason.

The scientific literature identifies unexpected foreign bodies such as portable heaters, toothbrushes and a shoehorn inserted into the rectum (3). However, tools such as a screwdriver being inserted into the rectum have not previously been reported.

Most cases of foreign bodies in the rectum can be treated in the emergency department by removing the object transanally (5). Spinal or pudendum anaesthesia may be necessary for these kinds of patients as it relaxes pelvic muscles and helps the patient push the object toward the anus and also reduces the patient's pain. Although multiple attempts to remove the object, or a lack of cooperation from the patient may result in the object moving further in or perforation of the rectum and/or anal sphincter. In such cases a laparotomy under general anaesthesia may be required (3).

\section{CONCLUSION}

Physicians should consider foreign objects in the rectum of patients with abdominal pain, especially when there is a history of psychological issues. In eastern and Islamic countries, unusual sexual toys may be present in the rectum, and are more dangerous because they might have sharp edges that may injury the rectum or bowel. If a foreign object is found impaled during a rectal examination, repeated rectal examinations should be avoided. The use of anaesthesia may be a safer method of aiding in the removal of the foreign object and thereby decreasing the potential for further injury, especially when the patient presents to the emergency department some hours after the object is found to be stuck by the patient.

\section{CONFLICT OF INTEREST}

The authors declare they have no conflict of interest.

\section{REFERENCES}

1. Goldberg JE, Steele SR, Rectal Foreign Bodies. Surg Clin N Am 2010; 90: 173-184.

2. Lake JP, Essani R, Petrone P, et al. Management of retained colorectal foreign bodies: predictors of operative intervention. Dis Colon Rectum 2004; 47: 1694.

3. Jung EJ, Ryu CG, Kim G, Hwang DY. Impaction of a Foreign Body in the Rectum by Improper Use of a (Electronic) Massager: A Case Report J Korean Soc Coloproctol 2010; 26(4): 298-301.

4. Yacobi Y, Tsivian A, Sidi AA. Emergent and surgical interventions for injuries associated with eroticism: a review. J Trauma 2007; 62: 1522-30.

5. Berghoff KR, Franklin ME Jr. Laparoscopicassisted rectal foreign body removal: report of a case. Dis Colon Rectum 2005; 48: 1975-7. 